\title{
Predictive Prefetching of Context-Aware Information in Mobile Networks
}

\author{
In Seon Choi ${ }^{1}$ and Gi Hwan $\mathrm{Cho}^{2}$ \\ ${ }^{1}$ Dept. of Computer Statistics \& Info., Chonbuk National Univ., Korea \\ ischoi@dcs.chonbuk.ac.kr \\ ${ }^{2}$ Div. of Electro. and Info. Eng. (Center for LBS Technology) Chonbuk National Univ., Korea \\ ghcho@dcs. chonbuk.ac.kr
}

\begin{abstract}
This paper presents a mobility prediction method for context-aware service in mobile networks. It aims to reduce the latency time to get the refreshed information appropriated to the current location of mobile users. The proposed method is to effectively limit the prefetched information into the most next location context. It makes use of the mobile reference count and the residence time that stands for the mobile user's visiting characteristics in any cell area. Then it also considers the information usability in order to further predict the prefetching candidates. In the numerical results, the proposed method is less latency time than that of the previous schemes to prefetch information matched to user's current location.
\end{abstract}

\section{Introduction}

Current trends in hardware and software development are making available powerful portable computing devices that communicate through wireless links with other mobile or fixed devices. At the same time, sensor technologies are going to be developed to allow these devices to keep track of their context (e.g., the Global Positioning System (GPS) for geographical position). As a consequence, there is an increasing interest in context-aware services, i.e. applications that modulate their behavior depending on the current context, where context usually means current time and location but it could also include a kind of available devices, the people surrounding the user, etc. For this kind of service, context changes may occur (e.g., user mobility) while an information request is still "active" (i.e., the user is still interested in the corresponding answer). Hence a context-aware information service must be able to provide a response appropriate to the user's current context, and to refresh a previously provided response if the corresponding request is still active after a change of context that invalidates the old response.

Technologies applied in existent network environment have a lot of restrictions to apply directly to wireless networks $[1,2]$. Namely, a mobile information service requires quick context aware conversion in movement, so the mobile user must get new information when moved to new location. The low bandwidth, high latency, traffic, and frequent connection due to the inherent characteristics of mobile networks are remained the obstacles to mobile users. Therefore, it is required to find a solution by utilizing the existing bandwidth instead of increasing the bandwidth that would cause 
additional expense. To resolve this, the most prominent method is how to prefetch information which is reflecting the user's current context.

The basic idea is to prefetch a set of information in advance and use them to accommodate mobile user's property. But a unsophisticated prefetching has the flaw that needs lots of memory and spends much computing time. So, to improve the performance of mobile information service, it is important to improve the prediction level of prefetching zone in order to give maximum effectiveness.

In this paper we propose location prediction method for context-aware information in mobile networks, which reduces the latency time to get the refreshed information appropriated to the current location of mobile users. The proposed scheme is based on high visiting frequency and residence time about space and user's data usability about information of the server.

The rest of the paper is organized as follows. In Section 2, an overview of related work is presented. In Section 3, a data usability model is presented and a predictive prefetching scheme is formalized with the mobility model and accumulated information. Using a simulation, numerical results are shown in Section 4. Finally a conclusion is given in Section 5 .

\section{Related Work}

Jiang and Kleinrock [3] proposed an adaptive network prefetching scheme. This scheme predicts the files' future access probabilities based on the access history and the network condition. The scheme allows the prefetching of a file only if the access probability of the file is greater than a function of the system bandwidth, delay and retrieval time.

Dar et al. [4] proposed to invalidate the set of data that is semantically furthest away from the current user context. This includes the current location, but also moving behaviors like speed, direction of the user. Ye et al. [5] makes use of predefined routes to detect the regions of interest for which data is required. In such a way, they have location information for the whole ongoing trip and do not have to compute the target areas while on the move.

Cho [1] suggested a prefetching approach by considering the speed and moving direction of the mobile user. The speed provides about the velocity with which a user changes locations. Moreover, the size of the user's area is largely dependent on the speed. Whenever the user crosses the borders of the current zone, new prefetching zones is computed. Depending on the speed in the moment that the user leaves the scope of a zone, the new one considers more or less adjacent network cells.

Choi [2] proposed prefetching scheme to append a time-based function to a frequency-based function. It makes use of the prefetching zone that reflects the user's mobility speed and direction, and the reference count that stands for the user's visiting frequency to given area. Then it considers the residence time, in order to further predict the prefetching candidates. But, if the number of prefetched information is full or the utilization rate of the prefetched information is low, wireless resource will be wasted such as bandwidth, etc. To avoid wasting resource, it is important that users prefetch the information with high frequency of use. 
This paper presents a progressively refined scheme, named in Data Usability Prefetching (DUP). The prefetching area is firstly limited with the locality of user's mobility pattern. It then is further confined with the information's usability behavior such as the information usability at a given cell area.

\section{DUP Prefetching Scheme}

This scheme is based on three prefetching models: The first is the spatial locality, which considers visiting frequency for prefetching. The second is the temporal locality which counts how many times should be residence in an area. The last is the data usability, which counts the user's interest of any data items.

\subsection{Prefetching System Architecture}

Fig. 1 shows the mobile information services system architecture. When a mobile user demands information, the context-aware information acquiring process is composed of step (1) (7). The requested information links to a server from the mobile device through the base station. The server is transmitting data items stored in database to mobile user. Information about interest and popularity as well as user's information updates and is kept in database. With applying prefetching in this environment, VPA (Virtual Prefetching Area) and APA (Actual Prefetching Area) take part in step A. In this way, we do to get information which a user is going to need in advance.

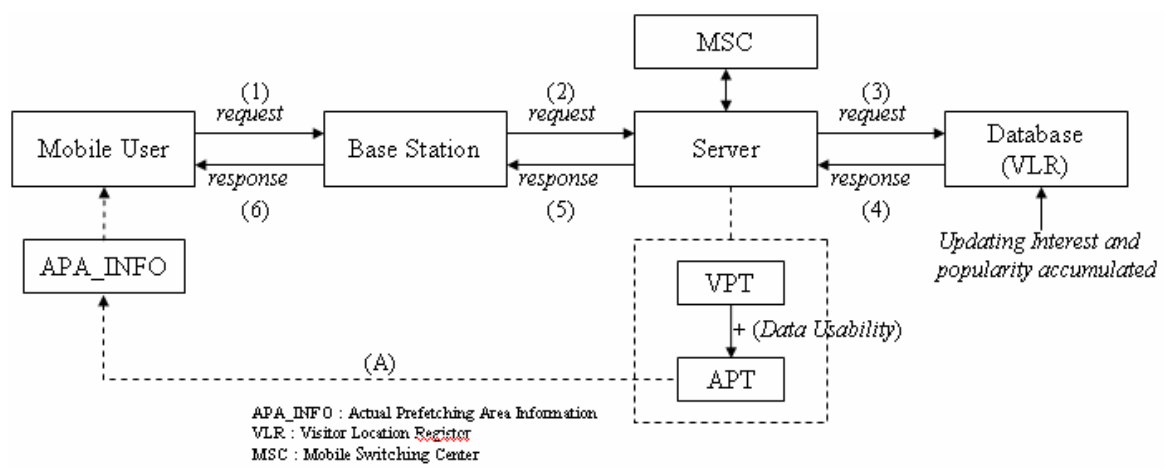

Fig. 1. DUP Prefetching System Architecture

VPA is virtual prefetching area derived from velocity-based prefetching, which is applied to accumulated frequency [1]. We can get the APA of real prefetching area by applying information usability in VPA. Information usability indicates access frequency about each data item of the server. We use information usability to prefetch information that has high frequency of use. Thus, in case of user revisit the same place we should be considered data usability. 
For prefetching a user's move pattern is assumed in the following. First, a user visits a location in which the user interested repeatedly in mobile environment. Second, revisit rate would be high on the location many users visited. If the user visits a location frequently, not only interest but also popularity must be considered. The interest indicates individual tendency and the popularity shows general tendency about any information.

\subsection{Spatial and Temporal Locality Based on Prefetching}

A frequency-based function $[1,2,6]$ is utilized by applying the reference count with the spatial locality. The reference count stands for the visiting frequency of each cell area, which reflects to the user's moving pattern. The following notations are used in our description:

$S_{\text {area }}$ : The velocity base prefetching, $r c:$ Reference counts of cell,

$f:$ Frequency, $N_{v e l}$ : The number of total cell established by velocity,

$r c w$ : Critical weight of the reference count $[0,1], i:$ Any cell number.

To make an effective decision of prefetching area, a prefetching threshold can be defined based on the frequency function. It would be applied to reduce prefetching area, so, with space-based criteria. Space-based prefetching threshold value, $P T_{s}$, is calculated as following.

$$
P T_{s}=\left(\frac{1}{N_{v e l}} \sum_{i \in S_{\text {area }}} r c(i) \cdot f(i)\right) \cdot r c w
$$

In addition to the frequency-based function, a time-based function can be utilized to minimize the number of prefetching area [2]. When observed user's moving pattern, free cell can be used only in path. However, in case of used temporal locality, information for needless free cell may not be prefetch. That is, if user was old retention time in an area, this means that there are a lot of need information in the area. We suppose for mean residence time $T_{i}$ in free cell. Accordingly, the time function can be written as

$$
T_{i}=\frac{\sum_{j=1}^{n} \operatorname{sum}_{j}}{r c}
$$

Where $n$ is the reference count of arbitrary cell and $s_{u m}$ is the sum of the residence time whenever a mobile user visits. To make a prefetching area decision, we determine a prefetching threshold. The following notations are used in our description:

$R_{\text {area }}:$ The reference based prefetching, $T:$ Average residence time of any cell,

$N_{\text {ref }}$ : The number of total cell established by reference,

$r w$ : Critical weight of the residence time $[0,1]$.

Temporal-based prefetching critical value $P T_{t}$ is as following.

$$
P T_{t}=\left(\frac{1}{N_{\text {ref }}} \sum_{i \in R_{\text {area }}} T(i) \cdot f(i)\right) \cdot r w
$$




\subsection{Information Usability Based on Prefetching}

Generally, a user may want to be got faster interesting information than other information [7]. User's any information can be divided into high and low about interest and popularity. Also, a classification of private interest and general interest according to user's condition will be useful to form a service structure. User's information supposes that was accumulated to server like Fig. 3. Also, Fig. 2 shows separation of personal history and general history about any information. Personal history shows user's interest and general history displays user's popularity. We use this distinction by basis strategy of prefetching. We present the proportion used of information, which allows us to measure the worth of a data item when making a prefetch decision. The following notations are used in the presentation:

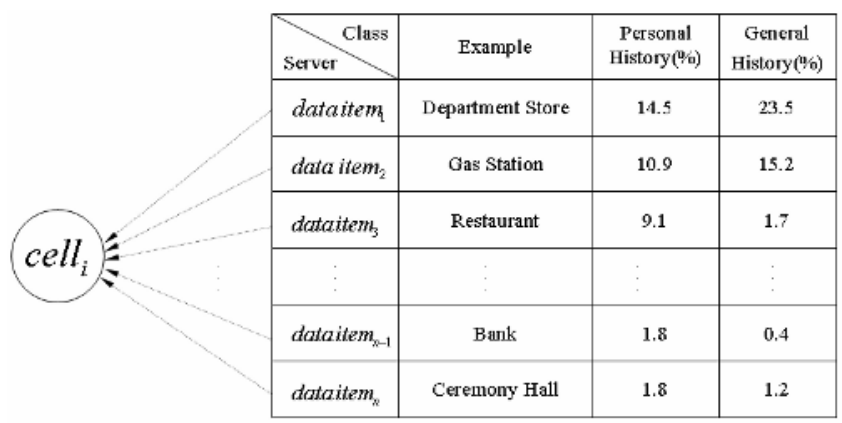

Fig. 2. Personal and general history of data items

The access probability of the data is used to identify the data to be prefetched. Intuitively, the ideal data item for prefetching should have a high access probability. Eq. (1) incorporates these factors to calculate the information usability of a certain cell $A$.

$$
A_{\text {usability }}=\sum_{i=1}^{N_{\text {tof }}} u_{i}
$$

In Eq. (1), the usability rate of the $i^{\text {th }}\left(1 \leq i \leq n_{\text {indi }}\right)$ data item is presented as follows.

$$
u_{i}=f_{a_{i}} / N_{t o t} * 100
$$

where $N_{\text {tot }}$ is number of total data items accessed in the database, $n_{\text {indi }}$ is number of data items in the database, $f_{a_{i}}$ is access frequency of data item $i, \sum f_{a}$ is access frequency accumulated of data items, $u_{i}$ is the usability rate of data item $i ;, \sum u$ is the usability rate accumulated of data items, $\bar{f}$ is the mean access frequency of data items.

The information usability decides which data item should be prefetched based on the access probability. The proposed scheme prefetches the data items that have 
higher $u_{i}$ based on the access probability. Therefore, information that is prefetched actually is data item with access frequency more than threshold. We prefetch information that data item's access frequency is more than average. Fig. 3 shows instance of information usability. Whole data item's number that is accessed in database is 52, and data item's number that belongs to database is 24. Frequency, accumulated frequency, information usability and accumulated information usability about data items shows in Fig. 3, and average frequency is 2.23 .

\begin{tabular}{|c|c|c|c|c|c|c|c|c|c|c|c|}
\hline Ord. & data item & $f_{0}$ & $\sum f_{0}$ & $\mathrm{u}_{\mathrm{i}}$ & $\sum \mathrm{u}$ & Ord & data item & $f_{0}$ & $\sum f_{0}$ & $\mathrm{u}_{\mathrm{i}}$ & $\sum \mathrm{u}$ \\
\hline 1 & Department Store & 8 & 8 & 14.5 & 14.5 & 12 & PC Room & 1 & 44 & 1.8 & 79.8 \\
\hline 2 & Gas Station & 6 & 14 & 10.9 & 25.4 & 12 & Court & 1 & 45 & 1.8 & 81.6 \\
\hline 3 & Restaurant & 5 & 19 & 9.1 & 34.5 & 12 & Beauty Salon & 1 & 46 & 1.8 & 83.4 \\
\hline 3 & Mart & 5 & 24 & 9.1 & 45.6 & 12 & Park & 1 & 47 & 1.8 & 85.2 \\
\hline 3 & Theater & 5 & 29 & 9.1 & 52.7 & 12 & Bakery & 1 & 48 & 1.8 & 87.0 \\
\hline 6 & Travel Agency & 3 & 32 & 5.5 & 58.2 & 12 & Police Station & 1 & 49 & 1.8 & 88.8 \\
\hline 7 & Zoo & 2 & 34 & 3.6 & 61.8 & 12 & Car Center & 1 & 50 & 1.8 & 90.6 \\
\hline 7 & City Hall & 2 & 36 & 3.6 & 65.4 & 12 & University & 1 & 51 & 1.8 & 92.4 \\
\hline 7 & Hotel & 2 & 38 & 3.6 & 69.0 & 12 & Health Club & 1 & 52 & 1.8 & 94.2 \\
\hline 7 & Airport & 2 & 40 & 3.6 & 72.6 & 12 & Playground & 1 & 53 & 1.8 & 96.0 \\
\hline 7 & Train Station & 2 & 42 & 3.6 & 76.2 & 12 & Bank & 1 & 54 & 1.8 & 97.8 \\
\hline 12 & Kindergarten & 1 & 43 & 1.8 & 78.0 & 12 & Ceremony Hall & 1 & 55 & 1.8 & 100.0 \\
\hline
\end{tabular}

Fig. 3. Example of information usability in the mobile server

The proposed scheme prefetches data items which have high usability based on the access probability. Accordingly, information that the specific person utilizes frequently in random cell is prefetched. If user is no wanted information in prefetched information, information that general person often utilizes prefetch. But, this paper considers personal history information only and general history information except. Fig. 4 shows the details of the acquiring process algorithm.

While ( $\mathrm{x}$ and y coordinate aren't the end)

obtain value from accumulated information;

// Spatial and Temporal Locality Model

$$
S_{\text {area }}=\left|\sqrt{V_{x^{2}}+V_{y^{2}}}\right| *\left|\left(\left|V_{x}\right|+\left|V_{y}\right|\right) / 2\right| ;
$$

$V_{x}$ and $V_{y}$ are mobile user's speed values. */

$R_{\text {area }} \leftarrow$ Select area with more than $P T_{s}$ (Eq. (2)) from $S_{\text {area }}$;

$T_{\text {area }} \leftarrow$ Select area with more than $P T_{t}$ (Eq. (3)) from $R_{\text {area }}$;

/* $T_{\text {area }}$ is the estimated time-based area. */

// Data Usability Model

for $\left(\mathrm{i}=0 ; \mathrm{i}<n_{\text {indi }} ; \mathrm{i}++\right)$

determine actual prefetching information with more than $P T_{d}$ using Eq. (5); /*PT : the DataUsability-based prefetching threshold value. */

End while

Fig. 4. Acquiring process algorithm 


\section{Performance Evaluation}

The DUP scheme is considered data usability. This is the improved prefetching method. In order to analyze its efficiency, the typical move scenario is presented. In the velocity-based mobility model, a user moves in a two-dimensional portion's area with the constant speed and direction during any given unit time period.

In this simulation, a mobile user is assumed to move around 25 by 25 portions. The user repeats process that move to position preserve of following destination 20 times according to given coordinate value beforehand. We establish virtual prefetching area with the different velocity in each move. A simulation has been done among the prefetching strategies with Velocity Prefetching (VP), Frequency Prefetching (FP), Spatial and Temporal(S\&TP) and DUP (Data Usability Prefetching). The numerical result has been measured in two aspects; the amount of prefetched portion information, and the utilization rate of prefetched portion information.

The simulator has been implemented using an event-based simulator CSIM [8]. A set of system parameters was given from the other works [6]: the bandwidth of wired line is assumed to very from $800 \mathrm{kbps}$ to $1.2 \mathrm{Mbps}$, the bandwidth of wireless medium is a tenth of that of wired line, the time of move detection is assumed to take $100 \mathrm{~ms}$. The mobility reference count has been obtained by accumulating for 30 days in simulation time.

\subsection{The Amount of Prefetched Portion Information}

With given the user mobility scenario, the number of prefetched portion information has been evaluated to show the communication and storage overhead of four prefetching strategies. Each simulation has been run based on the same system parameter, user move model and accumulated information of the server. Fig.5 shows the simulation result. It reports the total number of prefetched information, and the mean number of prefetched information. Proposed strategy DUP was shown improvement of performance about 0.5 when compared with S\&TP.

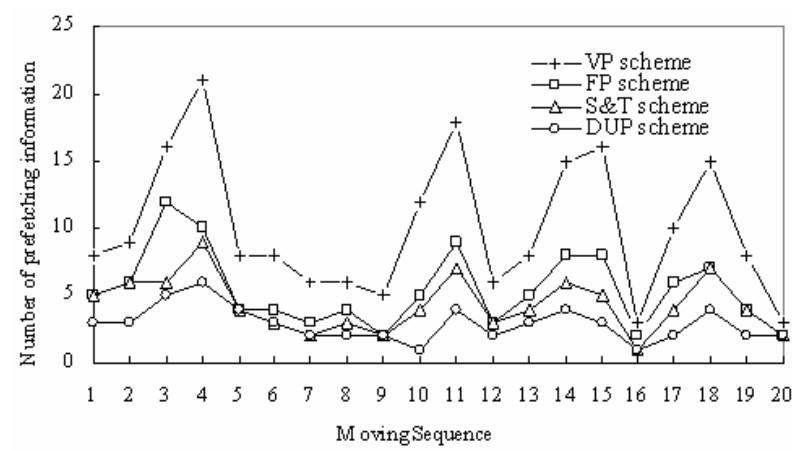

Fig. 5. The number of prefetched portion information 


\subsection{The Utilization Rate of Prefetched Information Portion}

Now, it is meaningful to figure out the utilization rate. The utilization rate can be achieved with the number of prefetched information actually participated for the user's location-aware service out of all the number of prefetched information. It just reflects the predictability degree for the given prefetching strategy. With the given user mobility model, the utilization rate with S\&TP is much better than that with FP; the former shows that over 0.858 of prefetched portion information is utilized for real service, while the later figures around 0.730 . With DUP, the rate is getting to 0.922 . Fig.6 summarizes the results of this experiment.

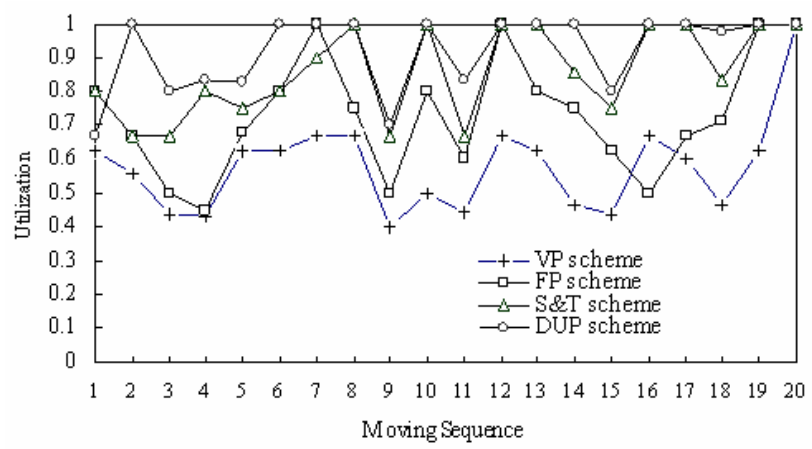

Fig. 6. Utilization rate of the prefetched portion information

\section{Conclusion}

The proposed scheme considers data usability based on information accumulated according to user mobility pattern. This has been achieved by defining the prefetching method with user's visiting frequency and residence time, and further with the data usability based on how many the information has been accessed. In addition to making smarter prefetch decisions, the scheme is designed to be adaptive, adjusting the prefetch rate based on current location and situation of mobile users. Simulation results verified that the proposed scheme can reduce the number of prefetched portion information and improve utilization rate of the prefetched portion information compared to the previous methods.

\section{References}

1. G.H. Cho, "Using Predictive Prefetching to Improve Location Awareness of Mobile Information Service," Lecture Notes in Computer Science, Vol. 2331, pp. 1128-1136, 2002

2. I.S. Choi, H.G. Lee and G.H. Cho, "Enhancing of the Prefetching Prediction for ContextAware Mobile Information Services, " Lecture Notes in Computer Science, Vol. 3794, pp. 1081-1087, 2005

3. Z. Jiang and L. Kleinrock, "An Adaptive Network Prefetch Scheme," IEEE Journal on Selected Areas in Communications, Vol. 16, no 3, pp. 1-11, April 1998 
4. S. Dar et. al, "Semantic Data Caching and Replacement," Proc. Of VLDB, 2002, pp. 330-341

5. Q. Ren and M.H. Dunham, "Using Semantic Caching to Manage Location Dependent Data in Mobile Computing," Proc. of MobiCom'00, 2000, pp. 210-221

6. S.M. Park, D.Y. Kim and G.H. Cho, "Improving prediction level of prefetching for locationaware mobile information Service," Future Generation Computer Systems, 2004, pp. 197-203

7. E.Valavanis et.al, "MobiShare: Sharing Context-Dependent Data \& Services from Mobile Sources," Proc. Of IEEE/WIC, 2003, pp.263-270

8. CSIM18 Simulation Engine, Mesquite Software Inc., 1997 